\title{
A associação entre variáveis sócio-demográficas e plumbemia em crianças
}

\author{
Denise Dascanio \\ Universidade Federal de São Carlos, São Carlos-SP, Brasil \\ Tania Gracy Martins do Valle \\ Universidade Estadual Paulista Júlio de Mesquita Filho, Bauru-SP, Brasil
}

\begin{abstract}
Resumo: Na busca de compreender a dimensão dos efeitos da plumbemia (nível de chumbo no sangue), este estudo investigou as variáveis sócio-demográficas de crianças residentes em uma região contaminada por chumbo. Para isto, foi realizada uma anamnese junto às mães de 40 crianças entre 7 e 13 anos, sendo 20 com baixa plumbemia $(<10 \mu \mathrm{g} / \mathrm{dl})$ e $20 \mathrm{com}$ alta plumbemia $(>20 \mu \mathrm{g} / \mathrm{dl})$. Os resultados foram categorizados e submetidos à análise estatística, que indicou diminuição da concentração de crianças na classe de alta plumbemia para baixa plumbemia no decorrer dos anos, sendo este declínio mais acentuado para os meninos do que para as meninas. Para as crianças com alta plumbemia, as mães relataram mais problemas de desenvolvimento, queixa escolar, problemas de saúde, de socialização e motricidade. Constatou-se ainda que as crianças filhas de mulheres cuja gestação ocorreu longe das fontes de contaminação apresentavam menor nível de plumbemia.
\end{abstract}

Palavras-chave: Distúrbios tóxicos. Desenvolvimento infantil. Nível socioeconômico.

\section{Association between demographic variables and blood lead in children}

\begin{abstract}
Seeking to understand the magnitude of blood lead effects, this study investigated socio-demographic variables of children from a lead contaminated area. Anamnesis was carried out with 40 mothers of children between 7 and 13 years of age, 20 with low blood lead level $(<10$ $\mu \mathrm{g} / \mathrm{dl})$ and 20 with high blood lead level (>20 $\mu \mathrm{g} / \mathrm{dl})$. Results indicate diminished concentration of children with high blood lead level over the years. This reduction is more pronounced in boys than in girls. Mothers of children with high blood lead levels report more development problems, complains at school, health, socialization and motor development problems. It was also evidenced that daughters of women whose pregnancy occurred far from sources of contamination, presented low blood lead level.
\end{abstract}

Keywords: Toxic disorders. Childhood development. Socioeconomic status.

\section{La asociación entre las variables sociodemográficas y el nivel de plomo en la sangre en los niños}

Resumen: Buscando comprender la dimensión de los efectos de la plumbemia (nivel de plomo en la sangre), este estudio investigó las variables socio-demográficas de niños residentes en una región contaminada por plomo. Para esto, fue realizada una anamnesis junto con las madres de 40 niños entre 7 y 13 años, siendo 20 con plumbemia baja $(<10 \mu \mathrm{g} / \mathrm{dl})$ y 20 con plumbemia alta $(>20$ $\mu \mathrm{g} / \mathrm{dl})$. Los resultados fueron categorizados y sometidos al análisis estadístico, el cual indicó una disminución de la concentración de niños en la clase de plumbemia alta para plumbemia baja en el recorrer de los años, siendo este declive más acentuado para los niños que para las niñas. Para los niños con plumbemia alta, las madres relataron más problemas de desarrollo, rendimiento escolar, problemas de salud, de socialización y motricidad. Además, se constató que los hijos de mujeres cuya gestación ocurrió lejos de la fuente de contaminación presentaron menor nivel de plumbemia.

Palabras clave: Trastornos tóxicos. Desarrollo infantil. Nivel socioeconómico. 
Episódios de contaminação ambiental por chumbo, embora estejam em declínio em países em desenvolvimento, continuam sendo freqüentes no Brasil, principalmente no que tange à contaminação crônica, altamente prejudicial à saúde infantil. Fato que evidencia a vulnerabilidade da fiscalização junto aos segmentos industriais, uma vez que as empresas são as principais responsáveis pelos focos de contaminação ambiental. Tendo em vista a importância global da contaminação por chumbo, o presente artigo visa compreender o problema sob os aspectos sócio-demográficos de crianças expostas às fontes de contaminação.

No Brasil não há legislação específica para o controle de chumbo em crianças, sendo então utilizados os índices propostos pelo Centers for Disease Control and Prevention (CDC, 1992) que afirma que a intoxicação por chumbo em níveis acima de $10 \mu \mathrm{g} / \mathrm{dl}$ no sangue pode causar alterações neurocomportamentais em crianças, tais como, déficits no desenvolvimento psicológico, desenvolvimento da linguagem e cognição, bem como alterações orgânicas que podem interferir na produção de hemoglobina, resultando em distúrbios gastrintestinais, hematológicos, perda de peso, retardo do crescimento e Linha de Burton (nome que se dá à coloração escura da gengiva, ocasionada pela contaminação por chumbo). Entretanto, a definição do nível tóxico de chumbo continua sendo pesquisada e tende a ser redefinida abaixo de $10 \mu \mathrm{g} / \mathrm{dl}$. Paoliello e Chasin (2001), discutindo sobre níveis aceitáveis de chumbo, afirmam que:

Nos últimos 20 anos, os padrões que definem os níveis aceitáveis de chumbo em crianças mudaram. Em 1975, o CDC, em Atlanta, recomendava uma concentração máxima de chumbo no sangue considerada segura para crianças igual a $30 \mu \mathrm{g} / \mathrm{dl}$. Dez anos mais tarde, esse nível diminuiu para $25 \mu \mathrm{g} / \mathrm{dl}$. Em 1991, baseado em evidências que mostravam a ocorrência de alguns efeitos adversos em níveis tão baixos quanto $10 \mu \mathrm{g} / \mathrm{dl}$, o CID e a Organização Mundial da Saúde (OMS) adotaram esse valor como nível limite oficial. Na Alemanha, 1996, a Comissão de Monitoramento Biológico também estabeleceu $10 \mu \mathrm{g} / \mathrm{dl}$ como nível aceitável de intoxicação (pp. 87-88).
Considerando a ampla gama de efeitos do chumbo no organismo e sua relação com as concentrações sanguíneas, suspeita-se que ainda não se tenha um limite seguro sobre sua concentração no sangue sem causar problemas nas crianças. Porém, a OMS (Organização Mundial de Saúde), o Centers for Disease Control and Prevention (CDC) e a American Conference of Governmental Industrial Hygienists (ACGIH) recomendam como aceitável uma concentração de chumbo no sangue das crianças menor que $10 \mu \mathrm{g} / \mathrm{dl}$. Isso significa que, segundo esses órgãos, a criança não é considerada intoxicada até níveis menores ou iguais a $9 \mu \mathrm{g} / \mathrm{dl}$, de acordo com o Centro de Vigilância Epidemiológica (CVE, 2002), que também afirma que aproximadamente $4 \%$ de todas as crianças do mundo têm níveis elevados de chumbo no sangue. Esta alta concentração parece estar relacionada aos locais onde tais crianças residem, ou seja, em áreas próximas a grande tráfego de automóveis, nas proximidades de minas, fundições de chumbo e fábricas de acumuladores. São exemplos disso as contaminações em solo em El Paso (México), na Suíça e no Brasil: Santo Amaro-BA, Paulínia-SP, Cubatão-SP, Caçapava-SP e Bauru-SP.

De acordo com as pesquisas do Centers for Disease Control (CDC, 1992), o chumbo pode ser absorvido por três vias distintas: a pele, o trato gastrintestinal e o sistema respiratório. Kahn, Kelly e Walker (1995) demonstram que as mulheres apresentam maior absorção oral de chumbo que os homens, dado que necessita de mais estudos, além disso, os meninos apresentam maior concentração sanguínea do metal do que as meninas. Acredita-se que esse fato decorra não de uma maior absorção pelos meninos, mas sim da maior atividade observada nas crianças do sexo masculino, que levam à boca com maior freqüência substâncias que podem estar contaminadas com o chumbo, como terra, pedras, excrementos, entre outros. Tong, McMichael e Baghurst (2000) encontraram em suas pesquisas que as crianças com desvantagem socioeconômica eram mais sensíveis aos efeitos do chumbo do que aquelas de status socioeconômico mais elevado, e que as meninas eram mais sensíveis aos efeitos do chumbo do que os meninos. 
A Agency for Toxic Substances and Disease Registry (ATSDR, 1990) aponta que os alimentos e bebidas ácidas, como picles, suco de tomate, de frutas e refrigerantes do tipo cola, cidra, quando embalados e estocados em recipientes mal esmaltados (latas amassadas) dissolvem o chumbo e contaminam-se. Outros componentes podem alterar a porcentagem de absorção do chumbo, por exemplo: alimentos cítricos e vitamina $\mathrm{C}$, que aumentam a acidez intestinal; vitamina $\mathrm{D}$, que aumenta a síntese de carregadores de cálcio e lactose, que podem aumentar a absorção do metal. Ao contrário, os íons de fósforo e magnésio, que competem pelos sítios de ligação dos carregadores da mucosa intestinal, bem como as fibras vegetais, que aumentam o peristaltismo, e a vitamina B podem diminuir a absorção do chumbo (Calabrese, 1978; Mahaffey, 1981). No adulto, cerca de $10 \%$ a $15 \%$ do chumbo ingerido é absorvido, sendo que sua maior parte é excretada in natura. Nas crianças e gestantes, a quantidade de chumbo absorvida chega a $40 \%$.

O sistema respiratório é a principal via de absorção do chumbo, porém algumas variáveis podem interferir: tamanho das partículas; concentração atmosférica do chumbo; ventilação individual, quanto maior for a ventilação, quantidade de ar inspirada e expirada por um indivíduo, maior será absorção; e composição química, compostos orgânicos de chumbo são mais facilmente absorvidos que compostos inorgânicos (Pimenta, 1988).

O papel do cálcio na absorção do chumbo é particularmente estudado. Acredita-se que os íons de chumbo e de cálcio ao serem absorvidos ocupam os mesmos sítios de ligação dos carregadores presentes na mucosa intestinal. Apesar de os carregadores apresentarem maior afinidade de ligação com o chumbo, uma dieta rica em cálcio irá sempre diminuir a absorção plúmbica, ou seja, a interação entre chumbo e cálcio é antagônica no processo de absorção intestinal (Wasserman, 1995; Ziegler, 1948). A relação entre chumbo e ferro também tem sido estudada, quando a dieta é pobre em ferro, ocorre uma estimulação dos carregadores da mucosa intestinal, de modo a absorver todo o ferro que está presente no trato gastrintestinal, fenômeno denominado hiperabsorção do ferro. Observa-se, nesses casos, uma hiperabsorção do chumbo (Bannon \& Chisolm, 2001; Bradman, Eskenazi, Sutton, Athanasoulis, \& Goldman, 2001; Wright, Shannon, Wright, \& Hu, 1999).

Segundo o MEDITEXT Medical Management (1998), o método mais comum na avaliação da contaminação por chumbo em crianças é a concentração de chumbo no sangue ( $\mathrm{Pb}-\mathrm{S})$, embora outros índices, tais como quantidade de chumbo nos ossos, nos cabelos ou nos dentes também estejam disponíveis. A concentração de chumbo no sangue reflete principalmente a história da exposição recente, enquanto o chumbo nos ossos representa um efeito cumulativo (Gulson, Mizon, Korsch, Palmer, \& Donnelly, 2003). Assim, por exemplo, uma criança que sempre residiu em uma região contaminada e outra que está exposta à contaminação há menos tempo terão $\mathrm{Pb}-\mathrm{S}$ semelhantes, embora a que sempre residiu na região provavelmente tenha mais chumbo depositado no tecido ósseo.

Inúmeros estudos têm fornecido evidências que a mobilização de chumbo dos ossos para o sangue é intensificada durante os períodos de maior liberação óssea, tais como crescimento rápido na infância, gravidez, lactação, menopausa, durante quadros de infecção, traumas, hiperparatireoidismo, desequilíbrios hormonais, entre outros (Moreira \& Moreira, 2004). Com o tempo, o chumbo concentra-se em grandes quantidades na medula óssea, diminuindo sensivelmente sua mobilidade (Pimenta, 1988).

A contaminação por chumbo, chamada comumente de saturnismo, acontece porque o chumbo, material estranho ao organismo humano, não é eliminado espontaneamente, acumulando-se nos ossos, no sangue e no sistema nervoso. Malta, Trigo e Cunha (2000) ressaltam ainda que, uma vez detectada a contaminação, o tratamento e ausência de chumbo no ambiente devem ser providenciados de imediato, visto que a eliminação deste metal é extremamente lenta, demorando até 10 anos para se efetivar.

As crianças e fetos são especialmente vulneráveis aos efeitos do chumbo devido à combinação de diversos fatores. A sensibilidade das crianças aos efeitos da exposição se dá, segundo Yule, Lansdown, Millar e Urbanowicz, (1981) porque: (a) o consumo por quilo de peso é maior do que nos adultos; (b) a absorção do chumbo pelo organismo da criança é maior 
do que pelo adulto; (c) estão em desenvolvimento rápido e constante, tornando-se mais vulneráveis aos efeitos do chumbo.

Na lactação ocorre substancial redistribuição de cálcio através da mobilização do cálcio ósseo (Nascimento, Izário-Filho, \& Baltazar, 2006). Estima-se que $5 \%$ da massa óssea seja mobilizada durante a lactação, dessa forma, o chumbo acumulado nos ossos da puérpera pode ser liberado para o sangue e excretado pelo leite materno. Trata-se de uma transferência ativa do sangue para o leite, a quantidade da substância foi estimada maior em até $300 \%$ no leite materno do que no sangue.

Nascimento e cols. (2006) realizaram um estudo associando diferentes níveis de chumbo no colostro humano. Os resultados apontaram que não houve significância estatística entre os valores de chumbo e as categorias: baixo peso e peso normal ao nascer e também com a idade gestacional categorizada a termo e pré-termo. Ettinger e cols. (2004) encontraram forte correlação entre os níveis de chumbo no leite de nutrizes um mês após o parto e os níveis de chumbo no sangue dessas mães e também no dos recém-nascidos. No referido estudo, realizado na Cidade do México, os autores mostraram que para cada $2 \mu \mathrm{g} / \mathrm{dl}$ de chumbo a mais no leite materno, havia um aumento de $0,82 \mu \mathrm{g} / \mathrm{dl} \mathrm{de}$ chumbo no sangue do lactante. Outros pesquisadores, como Lanphear, Dietrich, Auinger e Cox (2000) e Wasserman e cols. (1997) investigaram gestantes residentes em área contaminada por chumbo e acompanharam o desenvolvimento das crianças, constatando déficits nas habilidades motoras e escolares dessas.

Tong, Baghurst, Sawyer, Burns e McMichael (1998) realizaram um estudo para avaliar a reversibilidade dos efeitos do chumbo nas habilidades cognitivas em crianças, testando se o declínio das concentrações de chumbo no sangue, após os dois anos de idade, está associado a uma melhora cognitiva. Para isso, organizaram um estudo longitudinal com 375 crianças de uma comunidade rural e urbana de Port Pirie, no sul da Austrália. O desempenho intelectual das crianças foi avaliado aos 2 e 4 anos, apresentando média de contaminação por chumbo de 21,2 $\mu \mathrm{g} / \mathrm{dl}$, e numa terceira etapa entre 7 e 11 anos, cuja média para estas idades foi de 7,9 $\mu \mathrm{g} / \mathrm{dl}$, o instrumento de avaliação utilizado foi a Escala WISC. Os resultados alcançados indicaram que o déficit cognitivo associado com a exposição ambiental ao chumbo na infância parece ser somente parcialmente reversível com subseqüente declínio no nível de chumbo. Dietrich, Berger, Succop, Hammond e Bornschein (1993), também realizaram estudos com o enfoque supracitado, com a intenção de comparar o nível de chumbo no decorrer dos anos e sua influência no desenvolvimento de capacidades intelectuais. Esses autores destacam a persistência de déficits cognitivos no decorrer dos anos.

Kaufman (2001), em um artigo que revisa 26 estudos, alerta os pesquisadores à necessidade de se atentar para particularidades importantes, tais como: os níveis citados como altos e baixos de chumbo no organismo (a revisão mostrou que foi considerado baixo nível de chumbo no sangue valores entre 10 a $20 \mu \mathrm{g} / \mathrm{dl}$, alto mais de $20 \mu \mathrm{g} / \mathrm{dl}$ ); as diferentes formas de medição desses níveis (sangue, cabelos, dentes); controle estatístico de variáveis; cuidado ao relacionar estudos dos efeitos do chumbo no modelo animal para humanos; aspectos genéticos e do ambiente da criança. O autor aponta também que fatores como renda, nível de educação dos pais, QI materno, ocupação dos pais, número de membros na família, fatores estressantes do dia-adia, ansiedade materna, doença mental materna ou na família, pobreza, maus-tratos, negligência, tipos de estímulos intelectuais fornecidos à criança, históricos de problemas médicos são variáveis que podem interferir na cognição das crianças.

Na região de Bauru-SP, alvo deste estudo, uma fábrica de baterias situada em área urbana, rodeada por várias residências, totalizando em torno de 20 mil pessoas, contaminou a região com resíduos de chumbo. Foi também denunciado pelos órgãos locais de saúde que as verduras, ovos e leite produzidos em chácaras próximas ao local estavam contaminados com o metal. No ano de 2002 ocorreu a interdição da fábrica com a identificação de contaminação em uma área superior a $1 \mathrm{~km}^{2}$ e em 296 crianças de 0 a 12 anos de idade com nível de chumbo no sangue entre 10 e 90 $\mu \mathrm{g} / \mathrm{dl}$, bem como na fauna e flora ("Contaminação ambiental", 2004). 
Procurando compreender os prejuízos causados pela alta plumbemia, este artigo teve por objetivo investigar as variáveis sócio-demográficas de crianças residentes em uma região contaminada por chumbo em Bauru-SP, bem como, descrever os níveis de chumbo das crianças com alta plumbemia.

\section{Método}

\section{Participantes}

Participaram desta pesquisa mães de 40 crianças plumbímicas (que apresentam nível de chumbo no sangue), entre 7 e 13 anos de idade, de ambos os sexos, moradoras de bairros próximos (até $1 \mathrm{~km}$ ) à indústria de baterias que contaminou a região por chumbo (Núcleos Habitacionais da Periferia de Bauru-SP). As crianças foram agrupadas de acordo com a plumbemia: Grupo 1 (crianças com alta plumbemia) e Grupo 2 (crianças com plumbemia abaixo dos limites considerados tóxicos, a saber, $10 \mu \mathrm{g} / \mathrm{dl}$, aqui denominadas: baixa plumbemia).

\section{Material}

Foi utilizado um roteiro de anamnese simplificada para a coleta dos dados, dividido em sete tópicos: (a) Identificação da criança; (b) Dados da família; (c) Membros da família; (d) Tipo de moradia; (e) Desenvolvimento da criança; (f) Escolaridade; (g) Relacionamento familiar. Os dados foram agrupados em categorias de forma a possibilitar a correlação entre eles e a análise por percentis, após a realização da anamnese.

\section{Procedimento}

Foram utilizadas duas amostras: Grupo 1, crianças com alta plumbemia; Grupo 2, crianças com plumbemia abaixo dos limites considerados tóxicos, a saber, $10 \mu \mathrm{g} / \mathrm{dl}$, aqui denominadas: baixa plumbemia.

O Grupo 1, com 20 crianças com plumbemia acima de $10 \mu \mathrm{g} / \mathrm{dl}$, foi selecionado a partir da população já identificada de 316 crianças de 0 a 12 anos, atendidas no Centro de Psicologia Aplicada da Universidade Estadual Paulista Júlio de Mesquita Filho (UNESP-Bauru-SP), encaminhadas pela Diretoria Regional da Saúde (DIR X) desde maio de 2002. Deste total, em 2005, após coletas anuais de sangue, constou-se que 68 ainda apresentavam contaminação por chumbo acima do tolerável. A residência de cada uma das crianças foi localizada a partir do aceite e disponibilidade em participar da avaliação, resultando na amostra de 20 participantes. A amostra que compôs o Grupo 2 foi extraída de uma população de 301 crianças fornecidas pela DIR X com contaminação por chumbo abaixo de $10 \mu \mathrm{g} / \mathrm{dl}$, residentes na área de risco (até $1 \mathrm{~km}$ ) da fábrica de baterias. Desta população, foram selecionadas as crianças com plumbemia abaixo dos limites de quantificação ou menor que 10 $\mu \mathrm{g} / \mathrm{dl}$ para o pareamento com o Grupo 1 . As crianças do Grupo 2 foram emparelhadas ao Grupo 1 para os itens: idade, sexo, série e escola.

Todas as crianças desta pesquisa foram avaliadas por profissionais de análise laboratoriais do Instituto Adolfo Lutz, sob a responsabilidade da Diretoria Regional da Saúde (DIR X) de Bauru. A identificação do nível de plumbemia foi realizado a partir da técnica de espectrometria de absorção atômica, por forno de grafite. As análises sanguíneas ocorreram nos anos de 2002, 2004 e 2005 e as crianças intoxicadas por chumbo passam por avaliação psicológica anual no CPA (Centro de Psicologia Clínica) da UNESP-Bauru desde 2002, quando ocorreu a descoberta da contaminação, fazendo parte do Projeto "Atendimento emergencial a crianças de 0 a 12 anos contaminadas por chumbo: avaliação psicológica e acompanhamento". Todos os aspectos éticos devido à pesquisa com seres humanos foram respeitados de acordo com a Resolução 196/96 do Conselho Nacional de Saúde. A presente pesquisa foi aprovada pelo Comitê de Ética da Universidade Estadual Paulista Júlio de Mesquita Filho, campus de Bauru.

\section{Resultados}

Os resultados são apresentados de forma a contemplar os objetivos propostos neste estudo, sendo eles: investigar as variáveis sócio-demográficas de crianças residentes em uma região contaminada por chumbo em Bauru, tal qual, descrever os níveis de chumbo das crianças com alta plumbemia. Os dados foram separados no intuito de compreender as semelhanças e diferenças entre os grupos estudados, bem como analisar cada grupo em suas particularidades. Para isto, inicialmente procedeu-se à 
apresentação dos dados por meio de porcentagens, porém todos foram correlacionados entre si, Correlação de Spearman.

\section{Caracterização das crianças com alta plumbemia}

As crianças do Grupo 1, com alta plumbemia, foram agrupadas arbitrariamente em classes de chumbo I - de $10 \mu \mathrm{g} / \mathrm{dl}$ a $20 \mu \mathrm{g} / \mathrm{dl}$ e II - mais de 20 $\mu \mathrm{g} / \mathrm{dl}$; e de idade, A - de 7 a 9 anos e 11 meses, e B - de 10 a 13 anos. Por meio dessa distribuição observou-se que 8 crianças estavam na faixa etária $A$, das quais 3 são meninos e 5 são meninas e a maioria, 12 , estava concentrada na faixa etária $\mathrm{B}$, das quais 7 são meninos e 5 são meninas. Na faixa etária A, notou-se um equilíbrio entre a distribuição das crianças entre as classes I (4) e II (4) de plumbemia. Por outro lado, na faixa etária $\mathrm{B}$, a maioria das crianças concentravam-se na classe I (8) contra 4 crianças na classe II. Considerando a variável classe de plumbemia, percebeu-se que para a classe I existe um maior número de meninas (7) contra 5 meninos, já para a classe II ocorre o inverso, com maior número de meninos (5) em relação ao de meninas (3).

Os dados supracitados sugerem que quanto maior a faixa etária, menor a classe de chumbo em que a criança se encontra. Esta relação pode ser observada pela faixa etária B que tem 8 crianças (4 meninos e 4 meninas) na classe I e apenas 4 crianças (3 meninos e 1 menina) na classe II.

\section{Dados anamnésicos: descrição das crian- ças com alta e baixa plumbemia}

A partir dos dados da anamnese, organizouse a caracterização das crianças. Em relação à gestação das crianças do Grupo 1, dos 20 participantes, apenas um apresentou intercorrências: a mãe teve hipertireoidismo durante a gravidez. Todas as crianças nasceram a termo. A maioria (80\%) nasceu de parto normal e $20 \%$ de parto cesária.

Ao considerar os dados da anamnese para o Grupo 2, a maioria das gestações $(85 \%)$ ocorreu normalmente, enquanto que em $15 \%$ ocorreram intercorrências como anemia e toxoplasmose na mãe. Quanto ao tipo de nascimento, $100 \%$ tiveram parto a termo. Metade das crianças nasceu de parto natural e metade nasceu de cesariana.

Em relação aos outros itens da anamnese temos que: no Grupo 1, 85\% das famílias moravam em casa própria, $10 \%$ alugada e $5 \%$ em residência invadida. $\mathrm{O}$ tempo de residência no bairro para $70 \%$ das crianças variou entre 5 a 12 anos, 30\% delas moravam há mais de 10 anos, o que indica que desde a ocorrência da contaminação estão expostas ao chumbo, com média de 9,3 anos de residência no local. A maioria das crianças vivia com a mãe e o pai (85\%), $10 \%$ moravam com a mãe e o padrasto e apenas $5 \%$ somente com a mãe. As idades das mães na gestação variaram de 16 a 34 anos, sendo que $15 \%$ tinham 19 anos ou menos e $75 \%$ mais de 20 anos. O número de pessoas que residiam na casa variou de 3 a 8 , sendo que em $80 \%$ das famílias havia 5 pessoas ou mais na casa. $\mathrm{O}$ número de irmãos de cada criança variou de 1 a 5 , sendo que $25 \%$ era filho único.

Em relação ao tipo de moradia para o Grupo 2, $60 \%$ das famílias moravam em casa própria, $20 \%$ alugada e $20 \%$ invadida. Todas as crianças moravam na região onde ocorreu a contaminação há mais de 5 anos. O tempo variou entre 5 e 13 anos de residência no local, com média de 9,03 anos de residência. A maioria das crianças (75\%) residia com a mãe e o pai, $10 \%$ com a mãe e padrasto, $5 \%$ com pai e madrasta, $5 \%$ apenas com o pai e $5 \%$ somente com a mãe. As idades das mães na gestação variaram de 14 a 32 anos, sendo que 44,4\% tinham 19 anos ou menos, 55, 6\% tinham 20 anos ou mais. O número de pessoas que residiam na mesma casa variou de três a oito, sendo que em $85 \%$ das famílias havia 5 pessoas ou mais. O número de irmãos de cada criança variou de 1 a 5 , sendo que $10 \%$ era filho único.

Comparando os grupos, na Tabela 1 tem-se que o Grupo 1, alta plumbemia, apresentou mais problemas de saúde, socialização e problemas motores, além de maior porcentagem de queixa escolar quando comparado com o Grupo 2, que apresentou apenas em linguagem porcentagem maior de problemas, de acordo com o relato das mães. 
Tabela 1

Porcentagem dos participantes que apresentam problemas no desenvolvimento, segundo relato das mães

\begin{tabular}{cccccccc} 
Grupos & $\begin{array}{c}\mathbf{N}^{\mathbf{0}} \text { de } \\
\text { participantes }\end{array}$ & $\begin{array}{c}\text { Média Pb-S } \\
\text { em } \boldsymbol{\mu g} / \mathbf{d l}\end{array}$ & $\begin{array}{c}\text { Participantes } \\
\text { com queixa } \\
\text { escolar }\end{array}$ & \multicolumn{2}{c}{ Participantes com problemas de desenvolvimento } \\
\hline Grupo 1 & 20 & $19,9^{*}$ & $55 \%$ & $40 \%$ & $5 \%$ & $15 \%$ & $5 \%$ \\
Grupo 2 & 20 & $0,45^{* *}$ & $15 \%$ & $5 \%$ & $10 \%$ & $5 \%$ & $0 \%$ \\
\hline
\end{tabular}

* Para o cálculo da média, foram considerados os níveis de chumbo dos anos de 2002, 2004 e 2005.

**Nesse grupo, havia duas criança que apresentavam nível mensurável de plumbemia.

No tocante ao desenvolvimento das crianças do Grupo 1, os itens são assim apresentados: (a) saúde: $40 \%$ das crianças apresentaram algum problema além de doenças como catapora, caxumba e sarampo. Para estas crianças relatou-se problemas como anemia (2 crianças); uma criança teve convulsão aos 2 anos e outra possui uma doença imunológica que provoca a queda de plaquetas (púrpura trombo), todavia não há relato de seqüelas no desenvolvimento, além de $20 \%$ destes apresentarem alergia; (b) desenvolvimento motor e da linguagem: apenas uma criança $(5 \%)$ apresentou desenvolvimento irregular nestes itens como deambulação instável e fala desarticulada; (c) desenvolvimento social: $15 \%$ apresentaram comportamento anti-social como irritabilidade, agressividade e estranhamento com as pessoas. Em relação à queixa escolar, $55 \%$ das crianças apresentaram alguma queixa escolar, destes $35 \%$ estavam relacionadas à dificuldade de aprendizagem e atenção na sala de aula e $20 \%$ envolvia tanto aprendizagem e atenção, como agressividade com os pares e indisciplina na sala de aula.

Para as crianças do Grupo 2, os itens são assim apresentados: (a) saúde: $15 \%$ das crianças apresentam algum problema, como perda auditiva e alergia; (b) desenvolvimento motor: nenhuma criança apresentou problemas; (c) linguagem: em 10\% das crianças houve relatos de dificuldades, como atraso para o início da fala, vocabulário reduzido; (d) desenvolvimento social: apenas $5 \%$ das crianças apresentaram alguma irregularidade como timidez nas interações sociais. Quanto à queixa escolar, 15\% apresentaram agressividade e indisciplina na escola e problemas de atenção e aprendizagem.

Esses resultados quando comparados estatisticamente por meio do teste de Mann-Whitney , com $5 \%$ de significância, não apresentaram diferença significativas.

A Tabela 2 apresenta algumas diferenças percentuais entre os grupos, considerando-se características familiares. O Grupo 1 apresenta maior escolaridade dos pais, variável que pode contribuir para a qualidade da estimulação fornecida à criança, maior porcentagem de relato de bom relacionamento familiar, maior porcentagem de empregabilidade dos pais e maior tempo de residência no bairro. Para o Grupo 2, nota-se maior renda per capita e maior empregabilidade das mães em relação ao outro grupo.

Tabela 2

Caracterização das famílias do Grupo 1 e Grupo 2

\begin{tabular}{ccccccc}
\hline Grupos & \multicolumn{2}{c}{ Porcentagem } & & Média \\
& $\begin{array}{c}\text { Pais: ensino } \\
\text { fundamental incompleto }\end{array}$ & $\begin{array}{c}\text { Renda per } \\
\text { capita }\end{array}$ & $\begin{array}{c}\text { Bom relacionamento } \\
\text { familiar }\end{array}$ & $\begin{array}{c}\text { Empregabilidade } \\
\text { Pai }\end{array}$ & $\begin{array}{c}\text { Tempo de residência } \\
\text { no bairro }\end{array}$ \\
\hline Grupo 1 & $47,5 \%$ & 171,06 & $95 \%$ & $100 \%$ & $45 \%$ & 9,3 \\
Grupo 2 & $55 \%$ & 206,25 & $80 \%$ & $90 \%$ & $75 \%$ & 9,05 \\
\hline
\end{tabular}


Ao considerar o número absoluto de cuidadores que trabalham, tem-se que: 9 mães do Grupo 1 e 12 do Grupo 2 trabalhavam; dos pais, todos do Grupo $1 \mathrm{e}$ 9 do Grupo 2 trabalhavam. Do Grupo 2, 13 cuidadores trabalhavam, enquanto no Grupo 1, apenas 9. Quando o foco é a frequiência das crianças à creche, notou-se que no Grupo 1 a maioria delas (12) freqüentava, contra 5 do Grupo 2. Ao investigar os locais onde as crianças costumavam brincar, as respostas dos pais variaram entre quintal, rua, ou ambos. A Figura 1 apresenta o número absoluto do local onde as crianças brincavam. A maioria das crianças do Grupo 2 brincava no quintal, enquanto a maioria das crianças do Grupo 1 brincava na rua ou no quintal.

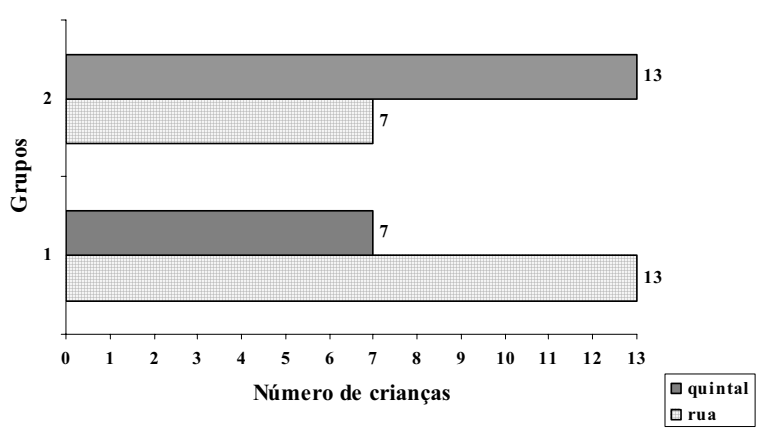

Figura 1. Comparação entre a freqüência de brincar na rua e no quintal entre o Grupo 1 e Grupo 2.

Em conjunto, os dados anamnésicos apontaram que: os pais do Grupo 1 tinham menor porcentagem de empregabilidade, as crianças freqüentavam a creche com maior frequiência e brincavam mais na rua; já no Grupo 2, os pais tinham porcentagem maior de empregabilidade, as crianças iam à creche com menor freqüência e brincavam com maior freqüência no quintal.

Comparou-se também o tempo de residência no bairro com as classes de chumbo (Classe I e II) para o grupo com alta plumbemia. A partir da idade das crianças, constatou-se que a maioria delas, 13 em cada grupo, sempre residiu na região, estando exposta às fontes de contaminação por chumbo desde a gestação. A Figura 2 apresenta as 13 crianças do Grupo 1 cuja gestação ocorreu na região, com a distribuição das crianças nas classes I e II de chumbo referente aos três anos em que elas foram avaliadas.

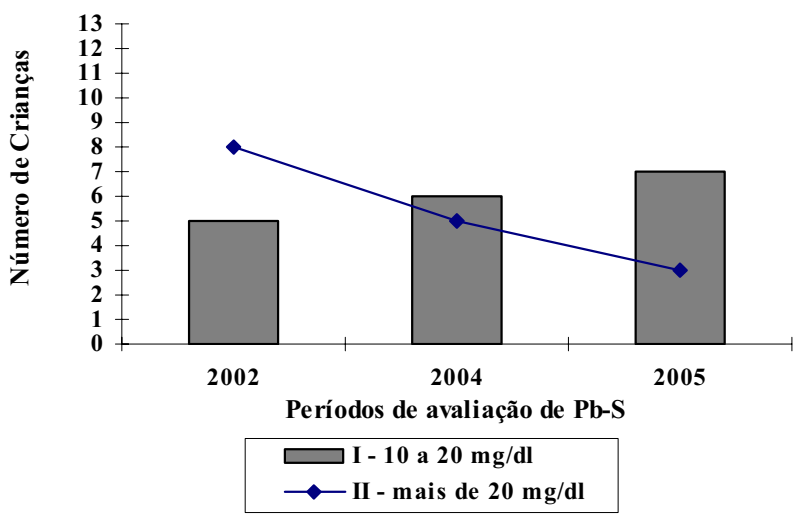

Figura 2. Crianças do Grupo 1 (13) cuja gestação ocorreu na região.

A Figura 3 apresenta a mesma distribuição para as sete crianças cuja gestação não ocorreu na região. É possível perceber pela linha de tendência do gráfico que houve diminuição da concentração de chumbo em crianças na classe II nas duas figuras.

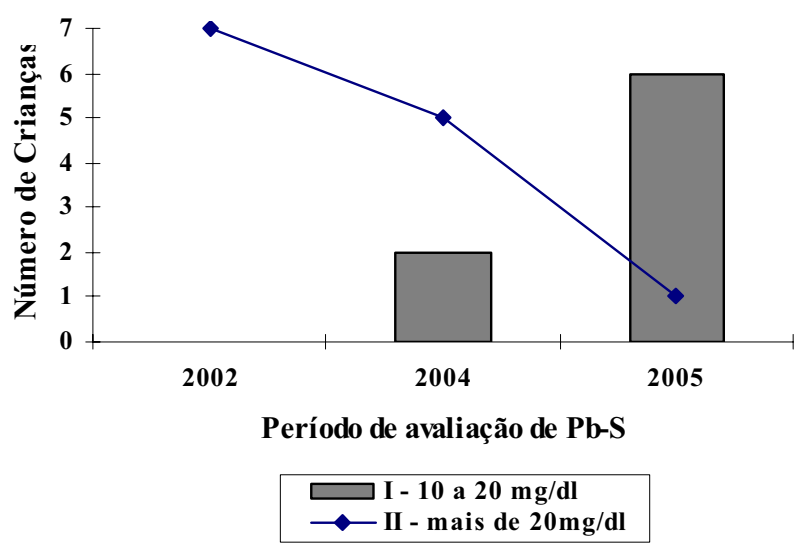

Figura 3. Crianças do Grupo 1 (7) cuja gestação não ocorreu na região.

\section{Discussão}

\section{Caracterização das crianças com alta plumbemia}

As crianças com alta plumbemia foram distribuídas em duas classes de níveis de contaminação para facilitar e pormenorizar a análise dos dados, de forma que, (a) classe I: de 10 a $20 \mu \mathrm{g} / \mathrm{dl}$; (b) classe II: 
maior que $20 \mu \mathrm{g} / \mathrm{dl}$. Essa divisão está de acordo com os achados de Kaufman (2001) que em seu estudo de revisão encontrou como baixo nível de chumbo no sangue os números entre 10 e $20 \mu \mathrm{g} / \mathrm{dl}$, e como altos níveis aqueles maiores que $20 \mu \mathrm{g} / \mathrm{dl}$.

Observou-se um declínio da concentração de crianças na classe II e um aumento de crianças na classe I, o que pode ser atribuído ao fato do diagnóstico ter sido realizado por meio da análise sanguínea, que é mais sensível para diagnosticar uma exposição recente ao metal, aliado ao fato de no decorrer dos anos, procedimentos de limpeza da região serem executados pela fábrica de baterias e vigilância sanitária, como raspagem da terra, limpeza das caixas de água e pavimentação de algumas ruas, juntamente com a eliminação da fonte primária de contaminação: interdição da fábrica de baterias ("Contaminação ambiental", 2004). Gulson e cols. (2003) salientaram em sua pesquisa que a plumbemia reflete uma história recente de exposição, conquanto não signifique ausência de contaminação, já que em função do tempo da exposição o chumbo acumula-se nos ossos.

Todavia, o declínio da concentração de chumbo não representa menor prejuízo a esta população, visto que a eliminação do chumbo no organismo infantil pode demorar até 10 anos para efetivar-se, de acordo com Malta, Trigo e Cunha (2000). Esse dado pode ser extremamente preocupante, uma vez que causa a falsa idéia de eliminação da contaminação, o que poderia contribuir para a negligência em relação a esta população, já que há uma aparente redução da contaminação e não há indícios de prejuízos visíveis em curto prazo nas crianças.

Quando se focaliza a distribuição entre as classes de chumbo por gênero, há um número maior de meninos que passaram para a classe I em comparação com as meninas, o que poderia indicar diferença entre os gêneros em relação à susceptibilidade aos efeitos do chumbo, como afirmam Tong e cols. (2000). Os dados também estão de acordo com os apontamentos de Kahn e cols. (1995) que perceberam concentração de chumbo maior para os meninos. Nota-se maior número de meninos do que de meninas na classe II de chumbo para o ano de 2002. Esse fato pode estar relacionado às práticas culturais que apregoam padrões de comportamentos distintos para meninos e meninas. Porém, não foi encontrada neste estudo significância estatística entre nível de chumbo e gênero. É provável que a associação entre gênero e quantidade de absorção de chumbo no sangue não seja tão facilmente medida, levando-se em consideração todas as variáveis que podem modular sua taxa de absorção, tais como fatores genéticos, peso, nutrição, escolaridade, como demarcam os estudos de Moreira e Moreira (2004).

Além da distribuição das crianças em classes de contaminação por chumbo, foi realizada divisão arbitrária por faixa etária: A (de 7 a 9 anos e 11 meses) e B (de 10 a 13 anos). Tal análise sugere que quanto maior a faixa etária, menor a classe de chumbo em que as crianças se encontram. Essa relação pode ser observada pela faixa etária $\mathrm{B}$, que tem 8 crianças (4 meninos e 4 meninas) na classe I e apenas 4 crianças ( 3 meninos e 1 menina) na classe II, dado que tem sustentação na literatura disponível. No estudo de Brody e cols. (1994), com idade variando de 1 ano até a faixa etária adulta, os resultados indicaram que os níveis de plumbemia foram mais elevados para as crianças mais jovens (entre 1 e 6 anos).

\section{Dados anamnésicos: descrição das crian- ças com alta e baixa plumbemia}

Todos os dados anamnésicos foram correlacionados entre si por meio da correlação de Spearman, sendo discutidos apenas aqueles que apontaram alguma correlação entre si ou que respondessem aos objetivos desta pesquisa. Ao focalizar o desenvolvimento das crianças observa-se que para aquelas com alta plumbemia havia mais relatos das mães de queixa escolar, problemas de saúde, de socialização e motor, embora sem significância estatística entre os grupos. Esses dados vão ao encontro dos achados de ATSDR (1990), que aponta prejuízos para as crianças contaminadas por chumbo. No grupo com baixa plumbemia, apenas em linguagem houve relatos de prejuízos maiores que para o grupo com alta plumbemia. Enquanto no Grupo 1 uma criança apresentou linguagem irregular, no Grupo 2 foram duas crianças.

Quando se analisa a caracterização familiar, nota-se que o Grupo 1 relatou melhor relacionamento familiar que o Grupo 2, resultado que pode 
estar vinculado ao fato de as crianças do Grupo 1 fazerem parte do projeto “Avaliação psicológica de crianças de 0 a 12 anos de idade contaminadas por chumbo", passando por freqüentes avaliações com profissionais da saúde e devido a isso apresentarem relato verbal mais adequado. Vale lembrar que a variável relacionamento familiar foi caracterizada como boa ou instável, sendo este último definido pela presença de brigas constantes entre a família.

$\mathrm{Na}$ tentativa de identificar fatores, arbitrariamente, que poderiam contribuir para que os grupos pareados apresentassem como principal diferença a plumbemia, avaliou-se a variável empregabilidade dos pais. Na seção Resultados, observou-se que no Grupo 1 a frequiência de empregabilidade dos cuidadores era menor que no Grupo 2, a freqüência à creche das crianças do Grupo 1 era maior que do Grupo 2. No Grupo 1, 11 mães permaneciam em casa, por serem donas de casa, o que poderia representar maior disponibilidade em cuidar da criança, todavia não é isso que se observa, pois a maioria das crianças deste grupo freqüentou a creche.

No Grupo 2, tem-se que 7 cuidadores (6 mães e 1 pai) permaneciam em casa, ou por serem donas de casa (4) ou por estarem desempregados (3). Todavia, as crianças deste grupo não freqüentaram a creche (15), o que parece incongruente, não se sabendo onde estas crianças ficavam durante o horário de trabalho dos pais. Uma hipótese é que elas poderiam permanecer aos cuidados de algum familiar em outra região, o que explicaria o fato de este grupo, que vive sob as mesmas condições do Grupo 1, não apresentar índice de contaminação por chumbo. Contudo, este dado não pode ser confirmado, uma vez que a anamnese não apresentou questionamentos para esclarecer onde as crianças ficavam enquanto os pais trabalhavam quando não freqüentavam a creche. Vale ressaltar que a variável freqüência à creche não se refere a um dado atual da criança, uma vez que todas estavam na primeira série ou posterior. A variável empregabilidade refere-se à condição atual dos pais, o que poderia não apresentar relação aparente, todavia, quando consideramos que a maioria dos cuidadores que permanecem em casa são donas de casa, refere-se a uma condição estável e não rotativa, como ocorre para aqueles que estão desempregados (três pais do Grupo 2 estavam desempregados).
Há ainda o fato de a maioria das mães do Grupo 2 trabalharem fora, o que pode indicar que no período gestacional passavam a maior parte do tempo em outra região, não estando expostas à contaminação, ao contrário das mães do Grupo 1, cuja maioria não trabalhava fora, logo, é possível que permanecessem mais tempo expostas às fontes de contaminação. Os estudos de Ettinger e cols. (2004), Nascimento e cols. (2006) e Wasserman e cols. (1997, 2003) apontam a transmissão da contaminação por chumbo via placenta, além de maior absorção plumbímica neste período (Pimenta, 1988; Yule, Lansdown, Millar, \& Urbanowicz, 1981).

Investigando-se a variável "local onde brinca", constatou-se que as crianças do Grupo 1 brincam mais na rua do que no quintal, comparadas com as do Grupo 2, estando assim mais susceptíveis à contaminação por chumbo, uma vez que o contato com a terra foi maior e tiveram mais oportunidade de colocar objetos na boca. Os pesquisadores Kahn e cols. (1995) apontaram relação entre maior número de atividades que favorecem a colocação na boca de objetos ou substância contaminada com chumbo e o nível de contaminação.

Neste estudo, vimos que as crianças de ambos os grupos apresentavam média de tempo de residência no local muito próxima, 9,3 anos para o Grupo 1, e 9,05 anos para o Grupo 2, sendo que 13 crianças em cada grupo já estavam expostas à poluição ambiental por chumbo desde seu período gestacional. Durante a gestação o chumbo pode contaminar o feto (Ettinger e cols. 2004; Wasserman e cols. 1997, 2003), assim $75 \%$ das crianças de cada grupo estariam expostas ao chumbo mesmo antes do nascimento. Isso aponta mais um item de pareamento entre os grupos, todavia, como já mencionado, as mães do Grupo 2 apresentavam maior empregabilidade que o Grupo 1, com isso permaneciam mais tempo longe da contaminação no período da gestação.

Dessa forma, foi possível comparar crianças expostas ao chumbo no período gestacional com crianças expostas ao chumbo no período pós-natal. Os dados da anamnese descritos nas Figuras 2 e 3 mostram que as crianças que residiam na região desde a gestação têm um declínio menor da concentração de chumbo no sangue em relação àquelas que residem 
na região apenas no pós-natal. Nas figuras supracitadas é possível observar declínio do número de crianças que ocupavam a classe II de chumbo (maior que $20 \mu \mathrm{g} / \mathrm{dl}$ ), porém, para aquelas cuja gestação ocorreu no local, a curva do gráfico apresenta declínio menor, indo ao encontro dos achados de Lanphear e cols. (2000) e Wasserman e cols. (1997, 2003), que encontraram déficits cognitivos em crianças que desde a gestação estavam expostas ao chumbo.

Mediante o resultado da anamnese, torna-se imperativo a busca pelas possíveis causas da diferença de contaminação por chumbo entre os grupos. Considerando que os dois grupos foram pareados para inúmeras variáveis, estavam expostos a condições e estímulos semelhantes, o que explicaria o fato de um grupo apresentar alta plumbemia e outro baixa? A literatura estudada aponta como fatores relevantes na absorção do chumbo pelo organismo a alimentação (Calabrese, 1978; Mahaffey e cols., 1981), o contato com as fontes de contaminação (CDC, 1992), a gestação e lactação (Wasserman e cols., 2003) e a capacidade ventilatória (Pimenta, 1988).

\section{Considerações finais}

A presente pesquisa avançou metodologicamente em relação a outras referenciadas em relação à inclusão de um grupo controle (baixa plumbemia), e controle de variáveis sócio-demográficas (Kaufman 2001). Todavia a metodologia utilizada para mensurar as variáveis demográficas pode não ter sido sensível para apontar as possíveis diferenças entre os grupos. Para estudos posteriores, aponta-se a necessidade de anamnese minuciosa, com questões referentes ao histórico alimentar da criança, histórico de estimulação proporcionada e pormenores do local onde permaneciam enquanto os pais trabalhavam. A resiliência à contaminação por chumbo das crianças do grupo com baixa plumbemia (Grupo 2) é uma variável a ser investigada, pois elas tiveram desenvolvimento pré e pós-natal em ambiente também precário no que refere às condições sócio-demográficas quando comparadas às crianças com alta plumbemia. Salienta-se que a terminologia resiliência está relacionada com processos psicossociais, que favorecem o desenvolvimento sadio do indivíduo, mesmo quando este se encontra frente a adversidades e problemas (Bianchini \& Dell'aglio, 2006), tal qual a contaminação por chumbo.

Fatos como esses motivam a tomada de providências no âmbito da pesquisa e intervenção. Partindo do pressuposto de que a contaminação por chumbo, em sua gravidade e impacto, é uma retratação da degradação ambiental resultante do processo industrial, são importantes estudos como este para que a sociedade reflita sobre quais seriam as estratégias de controle de comunidades, que têm seus alicerces (trabalho, moradia, escola) construídos em uma região de risco.

\section{Referências}

Agency for Toxic Substances and Disease Registry. (1990). Toxicological profile for lead. Atlanta, GA: Author.

Bannon, D. I., \& Chisolm, J. J., Jr. (2001). Anodic stripping voltammetry compared with graphite furnace atomic absorption spectrophotometry for blood lead analysis. Clinical Chemistry, 47, 1703-1704.

Bianchini, D. C. S., \& Dell'aglio, D. D. (2006). Processos de resiliência no contexto de hospitalização: Um estudo de caso. Paidéia (Ribeirão Preto), 16, 427-436.

Bradman, A., Eskenazi, B., Sutton, P., Athanasoulis, M., \& Goldman L. R. (2001). Iron deficiency associated with higher blood lead in children living in contaminated environments. Environmental Health Perspectives, 109, 1079-1084.

Brody, D. J., Pirkle, J. L., Kramer, R. A., Flegal, K. M., Matte, T. D., Gunter, E. W., \& Paschal, D. C. (1994). Blood lead levels in the US population. JAMA, 272, 277-283.

Calabrese, E. I. (1978). Pollutants and high-risk groups: The biological basis of increased human susceptibility to environmental and occupational pollutants. New York: John Wiley and Sons.

Centers for Disease Control and Prevention. (1992). Case studies environmental medicine: Lead toxicity. Recuperado em 10 maio 2004, de http:// wonder.cdc.gov/wonder/prevguid/p0000017/ p0000017.asp 
Centro de Vigilância Epidemiológica. (2002). Estratégias de abordagem para a exposição ambiental ao chumbo no Estado de São Paulo. Recuperado em 06 agosto 2002, de http:// www.cve.saude.sp.gov.br/htm/chumbo.htm

Contaminação ambiental por chumbo em Bauru. (2004). Jornal da Cidade. Recuperado em 06 junho 2004, de http://www.jcnetcom.br

Dietrich, K. N., Berger, O. G., Succop, P. A., Hammond, P. B., \& Bornschein, R. L. (1993). The developmental consequences of low to moderate prenatal and postnatal lead exposure: Intellectual attainment in the Cincinnati Lead Study Cohort following school entry. Neurotoxicology and Teratology, 44, 15-37.

Ettinger, A. S., Téllez-Rojo, M. M., Amarasiriwardena, C., Bellinger, D., Peterson, K. E., Schwartz, J., Hu, H., \& Hernández-Avila, M. (2004). Effect of breast milk lead on infant blood levels one month of age. Environmental Health Perspectives, 112, 1381-1385.

Gulson, B. L., Mizon, K. J., Korsch, M. J., Palmer J. M., \& Donnelly, J. B. (2003). Mobilization of lead from human bone during pregnancy and lactation: A summary of long term research. Science of the Total Environment, 303, 79-104.

Kahn, C. A., Kelly, P. C., \& Walker, W. O., Jr. (1995). Lead screening in children with attention deficit hyperactivity disorder and development delay. Clinical Pediatrics, 34, 498-501.

Kaufman, A. S. (2001). How dangerous are low (not moderate or high) doses of lead for children's intellectual development? Archives of Clinical Neuropsychology, 16, 403-431.

Lanphear, B. P., Dietrich, K., Auinger, P., \& Cox, C. (2000). Cognitive deficits associated with blood lead concentrations $<10 \mathrm{pg} / \mathrm{dL}$ in US children and adolescents. Public Health Reports, 115, 521-529.

Mahaffey, K. R. (1981). Nutritional factors in lead poisoning. Nutrition Review, 39, 13-19.

Malta, C. G. T., Trigo, L. A. S. C., \& Cunha, L. S. (2000). Saturnismo. Recuperado em 07 agosto 2002, de http://www.geocities.com/HotSprings/ Resort/4486/chumbo1.htm.
MEDITEXT Medical Management. (1998). In Tomes CPS System, Toxicology, occupational medicine and environmental serves [CD-ROM]. Englewood, CO: Micromede X.

Moreira, F. R., \& Moreira, J. C. (2004). Os efeitos do chumbo sobre o organismo humano e seu significado para a saúde. Revista Panamericana de Salud Pública, 15, 119-129.

Nascimento, L. F. C., Izário-Filho, H. J., Baltazar, E. O. (2006). Níveis de chumbo em colostro humano: Um estudo no Vale do Paraíba. Revista Brasileira de Saúde Materno Infantil, 6, 69-74.

Paoliello, M. M., Chasin A. A. M. (2001). Ecotoxilogia do chumbo e seus compostos. Cadernos de Referência Ambiental, 3, 87-88.

Pimenta, L. A. (1988). A história do programa municipal de saúde aos trabalhadores. In A. L. Pimenta \& D. Capistrano-Filho (Orgs.), Saúde do trabalhador (pp. 21-30). São Paulo: Hucitec.

Tong, S., Baghurst, P. A., Sawyer, M. G., Burns, J., \& McMichael, A. J. (1998). Declining blood lead levels and changes in cognitive function during childhood. The Port Pirie study. JAMA, 280, 1915-1919.

Tong, S., McMichael, A. J., \& Baghurst, P. A. (2000). Interactions between environmental lead exposure and sociodemographic factors on cognitive development. Archives of Environmental Health, 55, 330-335.

Wasserman, G. A. (1995). Effects of early lead exposure: Time to integrate and broaden our efforts. Neurotoxicology and Teratology, 17, 243-244.

Wasserman, G. A., Liu, X., Lolacono, N. J., FactorLitvak, P., Kline, J. K., Popovac D., Morina, N., Musabegovic, A., Vrenezi, N., Capuni-Paracka, S., Lekic, V., Preteni-Redjepi, E., Hadzialjevic, S., Slavkovich, V., \& Graziano, J. H. (1997). Lead exposure and intelligence in 7-year-old children: the Yugoslavia prospective study. Environmental Health Perspectives, 105, 956-962. 
Wasserman, G. A., Factor-Litvak, P., Liu, X., Todd, A. C., Kline, J. K, Slavkovich, V., Popovac D., \& Graziano, J. H. (2003). The relationship between blood lead, bone lead and child intelligence. Child Neuropsychology, 9, 22-34.

Wright, R. O., Shannon, M. W., Wright, R. J., \& Hu, H. (1999). Association between iron deficiency and low-lead poisoning in an urban primary care clinic. American Journal of Public Health, 89, 1049 - 1053.

Yule, Q., Lansdown, R. G., Millar, I. B., \& Urbanowicz, M. A. (1981). The relationship between blood-lead concentrations, intelligence and attainment in a school population: A pilot study. Developmental Medicine and Child Neurology, 23, 567-576.

Ziegler, E. J. (1948). Effect of material retained on the number 4 sieve on the compaction test of soil. Proceedings, Highway Research Board, 28, 409-414.

Artigo recebido em 07/06/2008.

Aceito para publicação em 17/12/2008.

As autoras agradecem a todos que participaram do projeto "Atendimento emergencial a crianças de 0 a 12 anos contaminadas por chumbo: avaliação psicológica e acompanhamento", na UNESP, campus Bauru-SP, e aqueles que contribuíram de alguma forma para a realização deste estudo: Cristiane Oliveira Alves, Rejane Cristina Baggio, Marta Regina Gonçalves Correia, Patrícia Kusumi, Telma Maria Ribeiro, Profa. Olga Maria Rolim Rodrigues, coordenadora do projeto, e Prof. Henrique Manuel Salgado que contribuiu com a análise estatística.

Endereço para correspondência:

Profa. Dra. Tânia Gracy Martins do Vale. Universidade Estadual Paulista Júlio de Mesquita Filho. Faculdade de Ciências. Departamento de Psicologia. Av. Eng. Luiz Edmundo Carrijo Coube, 14-01. CEP 17033-360. Bauru-SP, Brasil. E-mail: tgvalle@fc.unesp.br
Denise Dascanio é doutoranda pelo Programa de Pós-graduação em Psicologia do Centro de Educação e Ciências Humanas da Universidade Federal de São Carlos.

Tania Gracy Martins do Valle é Professora Titular da Faculdade de Ciências da Universidade Estadual Paulista Júlio de Mesquita Filho, campus Bauru-SP. 